Check for updates

Cite this: Nanoscale Adv., 2019, 1, 2979

\title{
Magnetically enhancing the Seebeck coefficient in ferrofluids
}

\author{
Thomas J. Salez, ${ }^{\text {ab }}$ Mansour Kouyaté, ${ }^{c}$ Cleber Filomeno, ${ }^{\text {cd }}$ Marco Bonetti, ${ }^{a}$ \\ Michel Roger, ${ }^{a}$ Gilles Demouchy, ${ }^{c e}$ Emmanuelle Dubois, (D) ${ }^{c}$ Régine Perzynski, (D) \\ Andrejs Cēbers (D) and Sawako Nakamae (D) *a
}

\begin{abstract}
The influence of the magnetic field on the Seebeck coefficient (Se) was investigated in dilute magnetic nanofluids (ferrofluids) composed of maghemite magnetic nanoparticles dispersed in dimethyl-sulfoxide (DMSO). A 25\% increase in the Se value was found when the external magnetic field was applied perpendicularly to the temperature gradient, reminiscent of an increase in the Soret coefficient $\left(S_{T}\right.$, concentration gradient) observed in the same fluids. In-depth analysis of experimental data, however, revealed that different mechanisms are responsible for the observed magneto-thermoelectric and -thermodiffusive phenomena. Possible physical and physico-chemical origins leading to the enhancement of the fluids' Seebeck coefficient are discussed.
\end{abstract}

Received 21st February 2019

Accepted 3rd June 2019

DOI: 10.1039/c9na00109c

rsc.li/nanoscale-advances

the last 20 years. Due to their superior thermal and electrical conductivities compared to their base-fluids, nanofluids first attracted attention as effective coolants in the 1990s. ${ }^{4}$ While much of the nanofluid research today still focuses on enhancing the fluids' thermal conductivity by adjusting various parameters such as nanoparticles' composition, ${ }^{5-7}$ coating materials and volume fraction, their application potential in other areas of renewable energy is also gaining momentum. ${ }^{8,9}$ For example, nanofluids have been explored for their optical properties (increased absorption) in solar collectors. ${ }^{\mathbf{1 0}, 11}$ The thermoelectric effects in liquid electrolytes containing charged colloidal particles and macro-molecules were also demonstrated both theoretically ${ }^{12-15}$ and experimentally, ${ }^{16,17}$ and the possibility of enhancing the thermoelectric energy conversion efficiency using charge-stabilised magnetic nanofluids (also known as ferrofluids) using thermo-electrochemical cells ${ }^{18,19}$ was reported very recently.

Thermo-electrochemical cells, or thermocells, produce an electrical current through redox reactions when two electrodes are maintained at different temperatures (thermogalvanic effect). Thermoelectric coefficients (equivalent to the Seebeck coefficient in solids) as high as a few $\mathrm{mV} \mathrm{K}^{-1}$ have been reported in liquid-containing thermocells, ${ }^{20}$ an order of magnitude larger than those of solid-state TE materials. We have recently demonstrated that the cumulative effects of thermo-electrically induced movements and distribution (the Soret effect) of nanoparticles and their electrostatic interactions with the electrodes can modify a thermocell's Seebeck coefficient. The net change in the Se can be either positive ${ }^{18}$ or negative, ${ }^{19}$ depending on the intricate balance between the NPs' surface charge, entropy of transfer and respective signs, and the nature of counterions present in the surrounding fluid. While the 
underlying physical and chemical mechanisms are far from being fully understood, these results paved a new direction in thermoelectric materials research based on nanofluids. In ferrofluids, it is quite well known that the Soret coefficient $\left(S_{\mathrm{T}}\right)$ and the diffusion coefficient $\left(D_{\mathrm{m}}\right)$ of ferrofluids depend on the strength and the direction of applied magnetic fields. ${ }^{21,22}$ For example, a marked increase in $S_{\mathrm{T}}$ is observed when the magnetic field is applied perpendicularly to the temperature gradient, while the opposite is true when applied in the parallel direction. ${ }^{21,23,24}$ Such magneto-thermodiffusion phenomena can be understood by taking into account the local magnetic field gradient within the fluid, ${ }^{24,25}$ and the existing theoretical model can reproduce experimental observations, provided that no magneto-convection occurs. ${ }^{24}$ Here, we examine the coupled Seebeck/Soret effects in ferrofluids under a magnetic field to determine to what extent one can take advantage of the magnetic nature of nanoparticles to control the thermoelectric potential of a thermocell. The value of Se is found to increase by as much as $25 \%$ in a dilute ferrofluid when a moderate magnetic field of $150 \mathrm{kA} \mathrm{m}^{-1}$ is applied perpendicularly to the temperature gradient inside a thermocell. To the best of our knowledge, this is the first experimental reporting of the enhancement of Se in ferrofluids by application of an external magnetic field.

In the following sections, we first describe the theoretical models used to analyse the effect of a magnetic field on the Seebeck coefficient in ferrofluids, ensued by the experimental approach used to measure the in-field Seebeck and Soret coefficients. The analysis and discussion of results expose the limit of our current understanding of magneto-thermoelectricity in ferrofluids, while highlighting possible physical origins that have been overlooked thus far and future research perspectives of ferrofluids.

\section{Theory under a homogeneous magnetic field}

In order to understand how a thermocell converts thermal energy into electricity, it is helpful to recognise two distinct states of operation; namely, the initial and the steady states as depicted in Fig. 1. The former refers to the instance immediately after the application of a temperature gradient across the thermocell. At this stage, various charged species, i.e., nanoparticles, redox couple molecules, ions, have not had enough time to diffuse and thus their concentration is still homogeneous throughout the cell. The latter is reached much later in time, when the thermodiffusion of all species has attained the Soret equilibrium, characterised by the cancelling of all particles' fluxes. The resulting Seebeck coefficient thus evolves over time from its initial value $\left(\mathrm{Se}^{\mathrm{ini}}\right)$ to the steady one $\left(\mathrm{Se}^{\mathrm{st}}\right)$. The detailed derivation of the Se dependence on different physical parameters such as the NP concentration and applied magnetic field is found in ref. 25. Here we present the salient features leading to the final expressions of the field dependence of $\mathrm{Se}^{\mathrm{ini}}$ and $\mathrm{Se}^{\mathrm{st}}$.
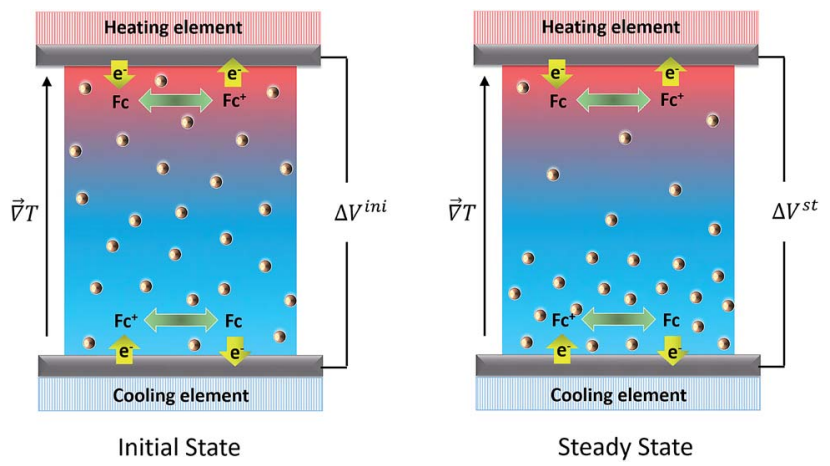

Fig. 1 Schematic images of an open-circuit thermocell containing redox molecules (e.g., $\mathrm{Fc}^{+}$(ferrocenium)/Fc (ferrocene)) and charged nanoparticles. Left panel: Initial state immediately after the application of a temperature gradient where NP concentration is still homogeneous. Right panel: Steady state established after the completion of thermodiffusive movements of all charged species. Note that in the open-circuit configuration, there is no electric current flowing into and from the thermocell. Therefore both reduction and oxidation reactions occur at the hot and cold electrodes. In this example, the charged NPs move toward the cold region of the fluid, corresponding to a positive $S_{\mathrm{T}}$. See the text for more explanation.

\subsection{Initial state}

The initial state Seebeck coefficient of a thermocell (Fig. 1 left panel) measured between two electrodes is expressed as: $:^{18,19}$

$$
\mathrm{Se}^{\mathrm{ini}}=-\frac{\Delta V^{\mathrm{ini}}}{\Delta T}=\frac{1}{e}[\underbrace{-\Delta s_{\mathrm{rc}}}_{\text {at electrode surface }}+\overbrace{\sum_{i} \frac{t_{i} \hat{S}_{i}}{\xi_{i}}}^{\text {bulk }}]
$$

$\Delta V$ and $\Delta T=T_{\mathrm{h}}-T_{\mathrm{c}}$ are the thermoelectric potential and temperature differences between the hot and cold electrodes, and $e$ is the elementary charge. The thermogalvanic term $\Delta s_{\mathrm{rc}}$ originates from the temperature dependent reaction entropy of the (reversible) redox couple at the electrode surfaces, which is expressed by the Nernst equation,

$$
\Delta s_{\mathrm{rc}}=\Delta s_{\mathrm{rc}}^{\circ}+\frac{k_{\mathrm{B}}}{\Delta T}\left[T_{\mathrm{h}} \ln \left(a_{\mathrm{h}}^{b_{\mathrm{b}}} a_{\mathrm{h}}^{b_{\mathrm{red}}}\right)-T_{\mathrm{c}} \ln \left(a_{\mathrm{c}}^{b_{\mathrm{ox}}} a_{\mathrm{c}}^{b_{\mathrm{red}}}\right)\right]
$$

$\Delta s_{\mathrm{rc}}$ is the standard reaction entropy of the redox couple, $k_{\mathrm{B}}$ is the Boltzmann constant and $a=\gamma \cdot n$ is the 'activity' defined as the product of the molar concentration $n$ of the reducing (oxidising) species and its activity coefficient $\gamma$. The latter depends on the ionic strength of the surrounding solution. ${ }^{26}$ The superscripts $b_{\text {ox }}$ and $b_{\text {red }}$ are defined by the redox chemical equation, such that

$$
b_{\mathrm{ox}} \cdot \mathrm{Ox}+e^{-}+b_{\text {red }} \cdot \mathrm{Red}=0
$$

In a closed-circuit operation mode, the magneto hydrodynamic effect is known to influence the electrical current of a electrochemical cell at very high magnetic fields (parallel or perpendicular to the electrode surface). ${ }^{27,28}$ However, to the best 
of our knowledge, no significant effect on the open-circuit thermogalvanic potential of a thermocell for a moderate magnetic field (below $800 \mathrm{kA} \mathrm{m}^{-1}$ or $1 \mathrm{~T}$ ) has been demonstrated. ${ }^{29}$

The second term in eqn (1) is related to the thermodiffusion in the bulk solution, summed over all charged species. $\hat{S}_{i}$ is the Eastman entropy of transfer of the $i$ th species (ions or nanoparticles). $\xi_{i}$ is the dynamical effective charge defined by:

$$
\xi_{i}=\frac{\zeta_{i} \mu_{\mathrm{elec}, i}}{e}
$$

where $\zeta_{i}$ and $\mu_{\text {elec, } i}$ are the friction coefficient and the electrophoretic mobility, respectively. For small, point-like ions, $\xi_{i}$ is simply the electrical charge number $z_{i}$. For colloidal nanoparticles $\xi_{\mathrm{NP}}$ is the dynamical effective charge ${ }^{24,30}$ which is close to, but not necessarily equal to, the static effective charge number $z_{\mathrm{NP}} . t_{i}$ is called the Hittorf number of the $i$ th species, which is equal to the fractional conductivity with respect to the total conductivity $\sigma_{\text {tot }}$, i.e., $\sigma_{i} / \sigma_{\text {tot }}$ where $\sigma_{i}$ is

$$
\sigma_{i}=\frac{z_{i} \xi_{i} e^{2} n_{i} D_{i}}{k_{\mathrm{B}} T}
$$

with $D_{i}$ being the mass diffusion coefficient. For superparamagnetic nanoparticles, $\hat{S}_{\mathrm{NP}}, \xi_{\mathrm{NP}}$ and $D_{\mathrm{NP}}$ all depend on the volume fraction of NPs $(\phi)$ and on the applied magnetic field $\vec{H}$.

When the interparticle repulsion is strong, as is the case in the ionically stabilized ferrofluids studied here, the $\phi$ dependence of the above listed parameters can be described well in terms of the isothermal osmotic compressibility $\chi$ ( $\left.\phi_{\text {eff }}\right)$ within the Carnahan-Starling hard-sphere model: ${ }^{31}$

$$
\chi\left(\phi_{\text {eff }}\right)=\frac{\left(1-\phi_{\text {eff }}\right)^{4}}{1+4 \phi_{\text {eff }}+4{\phi_{\text {eff }}}^{2}-4{\phi_{\text {eff }}}^{3}+\phi_{\text {eff }}{ }^{4}}
$$

$\phi_{\text {eff }}=\phi\left(d_{\mathrm{HS}} / d\right)^{3}$ is the effective NP volume fraction with an effective hard-sphere diameter $d_{\mathrm{HS}}=d+2 \lambda_{\mathrm{D}}$, with $\lambda_{\mathrm{D}}$ being the screening length.

The magnetic field dependence of $\hat{S}_{\mathrm{NP}}, \xi_{\mathrm{NP}}$ and $D_{\mathrm{NP}}$, on the other hand, is much less established. Here we use a meanfield model as commonly done in ferrofluids, ${ }^{32}$ where we consider that the nanoparticles are submitted to an effective field $\vec{H}_{\mathrm{e}}$ :

$$
\vec{H}_{\mathrm{e}}=\vec{H}+\lambda \vec{M}
$$

$\vec{H}$ is the macroscopic magnetic field, $\vec{M}$ is the local magnetisation of the bulk fluid and $\lambda$ is a dimensionless constant which is null for non-magnetic particles and equals to 0.33 for a uniformly magnetised medium (classical Lorentz result). ${ }^{33}$ For aqueous ferrofluids, $\lambda=0.22$ has been determined both experimentally $^{34-37}$ and numerically. ${ }^{38,39}$ Magnetisation of a ferrofluid composed of $n$ non-interacting NPs with $\vec{m}$ individual magnetic moment is given by ${ }^{33} \quad M=n m \mathscr{L}\left(x_{0}\right)$ where $\mathscr{L}\left(x_{0}\right)=\operatorname{coth}\left(x_{0}\right)-1 / x_{0}$ the classic Langevin function and $x_{0}=$ $\left(\mu_{0} m H\right) /\left(k_{\mathrm{B}} T\right)$ the Langevin parameter where $\mu_{0}$ is the vacuum permeability and $k_{\mathrm{B}}$ is the Boltzmann constant. In the framework of an effective field model, the Langevin parameter of an interacting NP system can be replaced by the effective Langevin parameter $x_{\mathrm{e}}$,

$$
x_{\mathrm{e}}=\frac{\mu_{0} m H_{\mathrm{e}}}{k_{\mathrm{B}} T}
$$

and must satisfy the self-consistency condition:

$$
x_{\mathrm{e}}=x_{0}+\lambda \psi_{\mathrm{dd}} \phi \mathscr{L}\left(x_{\mathrm{e}}\right)
$$

where $\lambda$ is the effective field parameter and $\psi_{\mathrm{dd}}=\mu_{0} m^{2} /\left(v_{\mathrm{NP}} k_{\mathrm{B}} T\right)$ is the dipolar interaction parameter, with $v_{\mathrm{NP}}$ being the volume of one nanoparticle. It represents the ratio between the dipoledipole interaction energy (i.e., physically contacting particles) and the thermal energy.

One can then obtain the analytical expressions of $\hat{S}_{\mathrm{NP}}, \xi_{\mathrm{NP}}$ and $D_{\mathrm{NP}}$ as a function of $\phi, \phi_{\text {eff }}$ and $H$ as follows. ${ }^{25}$

$$
\begin{aligned}
& \hat{S}_{\mathrm{NP}}\left(\phi, \phi_{\mathrm{eff}}, H\right)=\frac{\hat{S}_{\mathrm{NP}}^{0}+k_{\mathrm{B}}\left(S_{1}(\phi, H)-\delta_{\vec{\nabla} T \cdot \vec{H}} S_{2}(\phi, H)\right)}{\frac{1}{\chi\left(\phi_{\mathrm{eff}}\right)}-\alpha_{\lambda}(\phi, H)+\delta_{\vec{\nabla} T \cdot \vec{H}} \beta_{\lambda}(\phi, H)} \\
& \xi_{\mathrm{NP}}\left(\phi, \phi_{\mathrm{eff}}, H\right)=\frac{\xi_{\mathrm{NP}}^{0}}{\frac{1}{\chi\left(\phi_{\mathrm{eff}}\right)}-\alpha_{\lambda}(\phi, H)+\delta_{\vec{\nabla} T \cdot \vec{H}} \beta_{\lambda}(\phi, H)} \\
& D_{\mathrm{NP}}\left(\phi, \phi_{\mathrm{eff}}, H\right)= \\
& D_{\mathrm{NP}}^{0} \frac{\zeta^{0}}{\zeta(\phi)}\left[\frac{1}{\chi\left(\phi_{\mathrm{eff}}\right)}-\alpha_{\lambda}(\phi, H)+\delta_{\vec{\nabla} T \cdot \vec{H}} \beta_{\lambda}(\phi, H)\right]
\end{aligned}
$$

In $\lim _{H, \phi \rightarrow 0}, \hat{S}_{\mathrm{NP}}, \xi_{\mathrm{NP}}$ and $D_{\mathrm{NP}}$ become $\hat{S}_{\mathrm{NP}}^{0}, \xi_{\mathrm{NP}}^{0}$ and $D_{\mathrm{NP}}^{0}$, respectively, and $\delta_{\vec{\nabla}_{T \cdot \vec{H}}}=0$ for $\vec{\nabla} T \perp \vec{H}$ and $\delta_{\vec{\nabla} T \cdot \vec{H}}$ reaches 1 for $\vec{\nabla} T \| \vec{H} \cdot \zeta(\phi)$ takes into account the friction between the nanoparticles and the surrounding liquid. $\zeta^{0}=6 \pi \eta_{0} R_{\mathrm{H}}$ is the friction at $\phi=0$, with $\eta_{0}$ being the viscosity of the solvent and $R_{\mathrm{H}}$ the hydrodynamic radius of the nanoparticles. The parameters $\alpha_{\lambda}, \beta_{\lambda}, S_{1}$ and $S_{2}$ are defined as:

$$
\begin{gathered}
\alpha_{\lambda}(\phi, H)=\frac{\lambda \psi_{\mathrm{dd}} \phi \mathrm{L}^{2}\left(x_{\mathrm{e}}\right)}{1-\lambda \psi_{\mathrm{dd}} \phi \mathscr{L}^{\prime}\left(x_{\mathrm{e}}\right)} \\
\beta_{\lambda}(\phi, H)=\frac{\phi \psi_{\mathrm{dd}} \mathscr{L}^{2}\left(x_{\mathrm{e}}\right)}{\left[1-\lambda \psi_{\mathrm{dd}} \phi \mathscr{L}^{\prime}\left(x_{\mathrm{e}}\right)\right]\left[1+(1-\lambda) \psi_{\mathrm{dd}} \phi \mathscr{L}^{\prime}\left(x_{\mathrm{e}}\right)\right]} \\
S_{1}(\phi, H)=\ln \left(\frac{x_{\mathrm{e}}}{\sinh \left(x_{\mathrm{e}}\right)}\right)+\frac{x_{\mathrm{e}} \mathscr{L}\left(x_{\mathrm{e}}\right)}{1-\lambda \psi_{\mathrm{dd}} \phi \mathscr{L}^{\prime}\left(x_{\mathrm{e}}\right)} \\
S_{2}(\phi, H)=\beta_{\lambda}(\phi, H) \frac{x_{\mathrm{e}} \mathscr{L}^{\prime}\left(x_{\mathrm{e}}\right)}{\mathscr{L}\left(x_{\mathrm{e}}\right)}
\end{gathered}
$$

All these parameters tend to zero for $H=0$, or for $k_{\mathrm{B}} T \gg$ $\left(\mu_{0} m H_{\mathrm{e}}\right)$ (i.e. $\left.x_{\mathrm{e}} \rightarrow 0\right)$.

Noting that the ionic conductivity of the NPs and the ions is independent of the magnetic field up to the first order, the field dependent variation of $\operatorname{Se}^{\text {ini }}, \Delta \operatorname{Se}^{\text {ini }}(\phi, H)=\operatorname{Se}^{\text {ini }}(\phi, H)-$ $\operatorname{Se}^{\text {ini }}(\phi, 0)$, is given in ref. 25 as 


$$
\Delta \mathrm{Se}^{\mathrm{ini}}(\phi, H)=\frac{e z_{\mathrm{NP}} \phi D_{\mathrm{NP}}^{0}\left[S_{1}(\phi, H)-\delta \vec{\nabla}_{T \cdot \vec{H}} S_{2}(\phi, H)\right]}{\sigma_{\mathrm{tot}} T v_{\mathrm{NP}}}
$$

It needs to be mentioned that the term $S_{1}-S_{2}$ in eqn (17) is always positive and therefore the sign of $\mathrm{Se}^{\mathrm{ini}}$ under a homogeneous magnetic field is determined solely by the sign of $z_{\mathrm{NP}}$.

\subsection{Soret equilibrium}

The Soret equilibrium is reached once the thermodiffusive motions of all particles are completed inside the thermocell. At this stage we must distinguish two different types of Seebeck coefficients. The first one, $\mathrm{Se}^{\mathrm{Eq}}$, is determined from the thermoelectric voltage measured between the hot and cold electrodes $\left(\Delta V^{\mathrm{Eq}}\right):^{40}$

$$
\Delta V^{\mathrm{Eq}}=\mathrm{Se}^{\mathrm{Eq}} \Delta T=\frac{1}{e}\left[-\Delta S_{\mathrm{rc}}+\sum_{j} b_{j} \hat{S}_{j}\right] \Delta T
$$

It is summed over all species participating in the redox reaction (charged and neutral) and $b_{j}$ is defined by eqn (3). It should be noted that $\mathrm{Se}^{\mathrm{Eq}}$ only depends (directly) on the redox couple due to the rearrangement of the charged species in the solution which screens the electrodes from the internal electric field of the solution. ${ }^{25,41}$ The latter, however, can asymmetrically modify the ionic strength near the cold and hot electrodes and thus affect $\mathrm{Se}^{\mathrm{Eq}}$ indirectly through the thermogalvanic term, $\Delta s_{\text {rc }}$ (i.e., eqn (2)).

The second Seebeck coefficient, $\mathrm{Se}^{\mathrm{Eq}^{*}}$, is due to the internal thermoelectric field created within the bulk solution and away from the electrodes. While this value cannot be directly measured, it can be inferred from the Soret coefficient, $S_{\mathrm{T}}$. At the Soret equilibrium, the distribution gradient of nanoparticles $\vec{\nabla} n N P, S_{\mathrm{T}}$ and $\mathrm{Se}^{\mathrm{Eq}^{*}}$ are related to one another (up to the first-order) as: ${ }^{42-44}$

$$
\frac{\vec{\nabla} n_{\mathrm{NP}}}{n_{\mathrm{NP}}}=-S_{\mathrm{T}} \vec{\nabla} T ; \quad S_{\mathrm{T}}=\left(\hat{S}_{\mathrm{NP}}-\xi_{\mathrm{NP}} e \mathrm{Se}^{\mathrm{Eq}}\right) / k_{\mathrm{B}} T
$$

It can be shown from the particle flux equation that ${ }^{25}$

$$
\mathrm{Se}^{\mathrm{Eq}}=\frac{\sum_{i} z_{i} n_{i} \hat{S}_{i}}{e \sum_{i} z_{i} \xi_{i} n_{i}}
$$

where $\hat{S}_{\mathrm{NP}}$ and $\xi_{\mathrm{NP}}$ depend on $\phi, \phi_{\mathrm{eff}}$ and $H$ as described in the previous subsection. ${ }^{18}$

\section{Experimental}

\subsection{DMSO-based ferrofluids}

The ferrofluid (FF) samples used in this study are composed of maghemite $\left(\gamma-\mathrm{Fe}_{2} \mathrm{O}_{3}\right)$ nanoparticles dispersed in an acidic solution of DMSO (dimethyl sulfoxide), similar to those used in ref. 19. The nanoparticles were first synthesised in water using the well-known Massart technique, ${ }^{45}$ then transferred into DMSO. ${ }^{30}$
The NP mean diameter is $6.7 \mathrm{~nm}$ with a log-normal polydispersity of 0.38 . These NPs are $\mathrm{H}^{+}$-coated (with perchlorate counter-ions, $24 \mathrm{mM}$ free $\mathrm{ClO}_{4}{ }^{-}$) and thus positively charged. Under such ionic strength conditions, the interparticle interaction balance is repulsive (see ref. 44), and the second coefficient $A_{2}$ of the virial development of the osmotic pressure becomes positive. Here, $A_{2}$ $=12$ and thus $\phi_{\text {eff }}=3 \phi$, as attested by the diffusion coefficient measurements as a function of $\phi$ at $H=0$ in Fig. 3(a). These ferrofluids possess high $S_{\mathrm{T}}$ values, e.g., $1.1 \mathrm{~K}^{-1}$, at an NP volume fraction $(\phi)$ of $0.25 \%$. For the Seebeck coefficient measurements, a redox couple composed of ferrocene (Fc) (Aldrich, 98\% pure) and ferrocenium $\left(\mathrm{Fc}^{+}\right)\left(\mathrm{FcBF}_{4}\right.$ salt from Aldrich, technical grade) was added to the solution at $3 \mathrm{mM}$ each. The NPs' effective dynamic charge $\xi_{\mathrm{NP}} e \approx 30$ was determined by electrophoretic measurements, and is in close agreement with the value of 25 reported in similar DMSO-based ferrofluids. ${ }^{19}$

\subsection{Thermodiffusion measurements}

The Soret and the NP diffusion coefficients were determined using the Forced Rayleigh Scattering (FRS) technique ${ }^{46}$ with a pump-beam from a high power $\mathrm{Hg}$ arc lamp (modulated at 100 $\mathrm{Hz}$ ). The incident beam travels through a grid and is focused by a camera lens on the ferrofluid sample surface contained in an optical cell with a thickness of $\alpha=100 \mu \mathrm{m}$ and a height of several $\mathrm{mm}$. This imprints an optical grating image in the thermalized sample with a periodicity of $\Lambda=90 \mu \mathrm{m}$. Owing to the optical absorption by the NPs, a thermal grating with the same spatial modulation is then produced inside the fluid. This, in turn, induces the migration of NPs due to the Soret effect, resulting in a concentration grating of NPs. The migration can either be towards the hot regions or the cold regions, depending on the colloidal characteristics of the system. Here, the NPs migrate towards the cold region. Both the thermal and the concentration gratings are detected by the diffraction of a weakly absorbing test laser beam (He-Ne). The first order diffraction pattern formation of the test beam and its progressive destruction by NP diffusion when the pump beam is shut down are recorded. As the scales of the temporal evolution of temperature and of the NP concentration are different by several orders of magnitude they can be decoupled. This allows the determination of the Soret coefficient $S_{\mathrm{T}}$ in the steady-state condition and the mass diffusion coefficient $D_{\mathrm{m}}$ of the NPs. ${ }^{46}$ These coefficients are measured as a function of NPs' volume fraction $\phi$ between 0.25 and $4 \%$, while the magnetic field dependence is examined on a sample with $\phi=3.44 \%$ only. A uniform magnetic field up to $160 \mathrm{kA} \mathrm{m}^{-1}$ is provided using an electromagnet with the measurement cell plane lying either parallel or perpendicular to the field direction..$^{34}$ The colloidal stability of the ferrofluid (with $\phi=3.44 \%$ and without the redox couple) has been verified by in-field optical scattering methods ${ }^{47}$ up to $H=80 \mathrm{kA} \mathrm{m}^{-1}$ and within the time span of the FRS experiments (of the order of one hour).

\subsection{Seebeck coefficient and AC conductivity measurements}

The Seebeck coefficient and the AC ionic conductivity values are studied for two NP concentrations, $\phi=0.28$ and $1 \%$. A home- 
made cylindrical Teflon cell (as described in previous work ${ }^{\mathbf{1 8 4 8}}$ ) was used for the Seebeck coefficient measurements. The liquid sample is contained in the cell's central cavity of 6 $\mathrm{mm}$ in diameter and $6 \mathrm{~mm}$ in height (sample volume is 0.17 $\mathrm{cm}^{3}$ ). The cell is sealed at the top and the bottom by $10 \mathrm{~mm}$ diameter platinum electrodes (AlphaAesar, 99.99\% pure) squeezed tightly using $10 \mathrm{~mm}$ copper pieces. The electrode surface is cleaned with concentrated $\mathrm{HCl}$ (Sigma-Aldrich, 37 wt\%) and washed by ultrasonication in deionised water. The electrode surface area in contact with the liquid is $A \approx 0.28$ $\mathrm{cm}^{2}$. The Seebeck coefficient is determined from the opencircuit voltage $\Delta V$ (as shown in Fig. 1) between the top and bottom electrodes measured while the thermocell is heated from the top, which limits the natural convection of the ferrofluid. $\Delta V$ is measured using a high impedance electrometer (Keithley 6514) and the Seebeck coefficient is calculated via Se $=-\Delta V / \Delta T$. The experiments are carried out between 20 and $50{ }^{\circ} \mathrm{C}$ with a $\Delta T$ of 10 or $30 \mathrm{~K}$. The temperature inside the cell is stabilised within a few minutes after the gradient has been imposed at which point the initial $\Delta V^{\text {ini }}$ is recorded. The apparent steady state potential $\Delta V^{\text {st }}$ is reached after several hours (see Fig. 2). Note that we differentiate this apparent steady state Seebeck coefficient $\mathrm{Se}^{\mathrm{st}}$ from Se $\mathrm{eq}^{\mathrm{eq}}$ (corresponding to that of Soret equilibrium state) introduced earlier for reasons that will be made clear in the Results and discussion section below.

A horizontal, homogeneous magnetic field, i.e., perpendicular to the thermal gradient, between 0 and $400 \mathrm{kA} \mathrm{m}^{-1}$ is applied to the thermocell using an electromagnet (Bouhnik). The perpendicular field direction is chosen following the Soret coefficient measurements where a marked increase in $S_{\mathrm{T}}$ is usually detected under a perpendicular magnetic field ${ }^{\mathbf{2 4}}$ (see the Results and discussion section for more details). Each temperature step lasts between 8 and 24 hours to fully reach the apparent steady state, depending on the $H$ strength. Both $\mathrm{Se}^{\text {ini }}$ and $\mathrm{Se}^{\text {st }}$ are measured as a function of the magnetic field

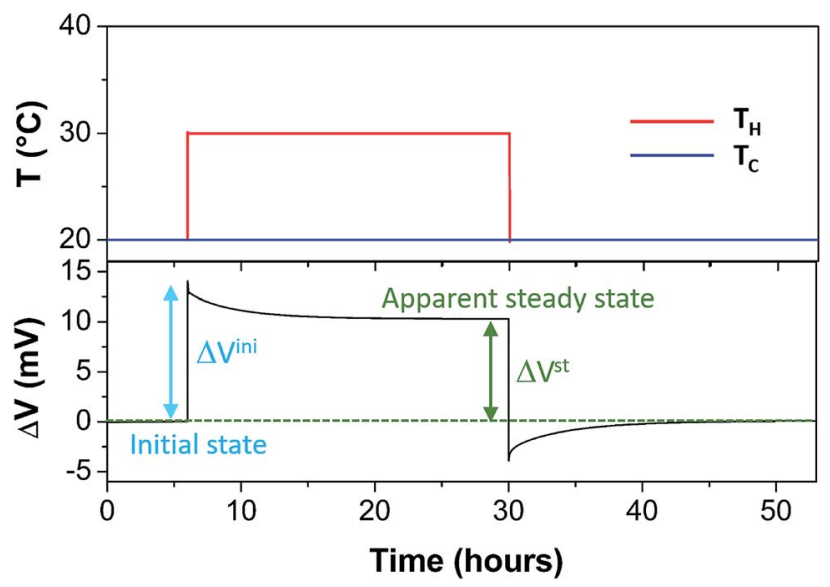

Fig. 2 Typical thermoelectric measurement. $T_{H}$ is the hot electrode temperature and $T_{\mathrm{c}}$, the cold electrode temperature. Here, the nanoparticle concentration is $0.28 \%$. An apparent steady state is reached after $\sim 15$ hours. See the text for more details. applied. The measurements are reproducible over several weeks with a low data dispersion.

The AC ionic conductivity measurements are also performed in the same thermocell using a precision LCR meter (HP 4284A) at $20 \mathrm{kHz}$, at which the out-of-phase component of the impedance becomes null. The total conductivity of the solution at 25 ${ }^{\circ} \mathrm{C}$ is determined to be $\sigma_{\text {tot }}=65 \mathrm{mS} \mathrm{m}^{-1}$ independent of the NP concentration (i.e., the ionic conductivity is dominated by the small counterions whose concentration was kept constant for all ferrofluids examined).

\section{Results and discussion}

\subsection{Thermodiffusion and Soret coefficient}

The NPs' diffusion coefficient $D_{\mathrm{NP}}$ and the Soret coefficient $S_{\mathrm{T}}$ measured as a function of $\phi$ in the absence of an applied magnetic field are shown in Fig. 3(a) and (b). The diffusion coefficient at the infinite dilution limit $D_{\mathrm{NP}}^{0}=D_{\mathrm{NP}}(\phi \rightarrow 0)$ and

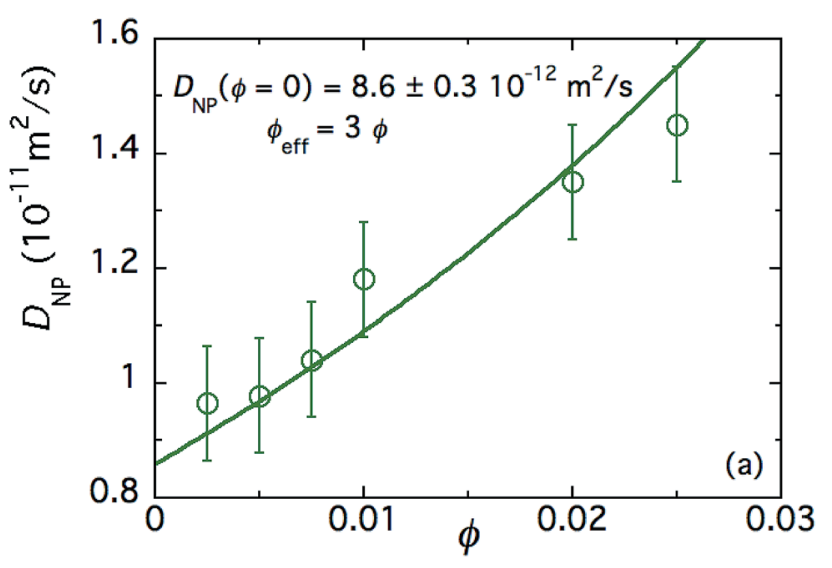

(a)

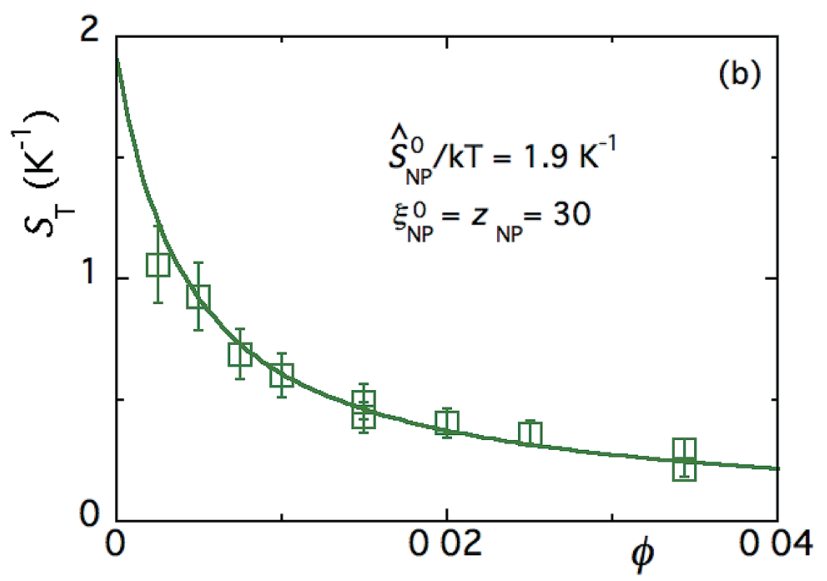

(b)

Fig. 3 (a) Diffusion coefficient as a function of NP concentration $\phi$ of FF-DMSO, measured at room temperature in the absence of a redox couple. The solid line is a fit to eqn (12) as a function of $\phi$, without a magnetic field. (b) Soret coefficient measured via the FRS technique as a function of NP concentration $(\phi)$, measured at room temperature in the absence of a redox couple. The solid line is a fit to eqn (19) as a function of $\phi$, without a magnetic field. 


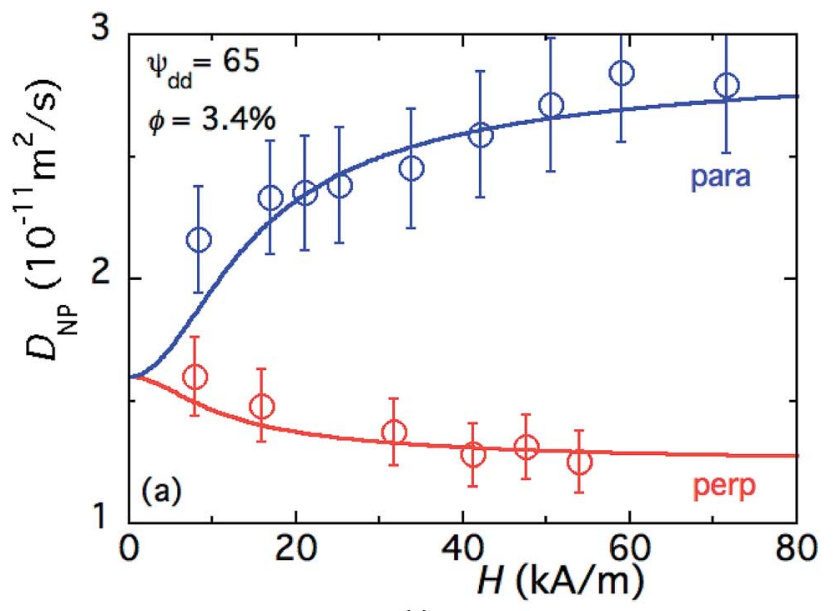

(a)

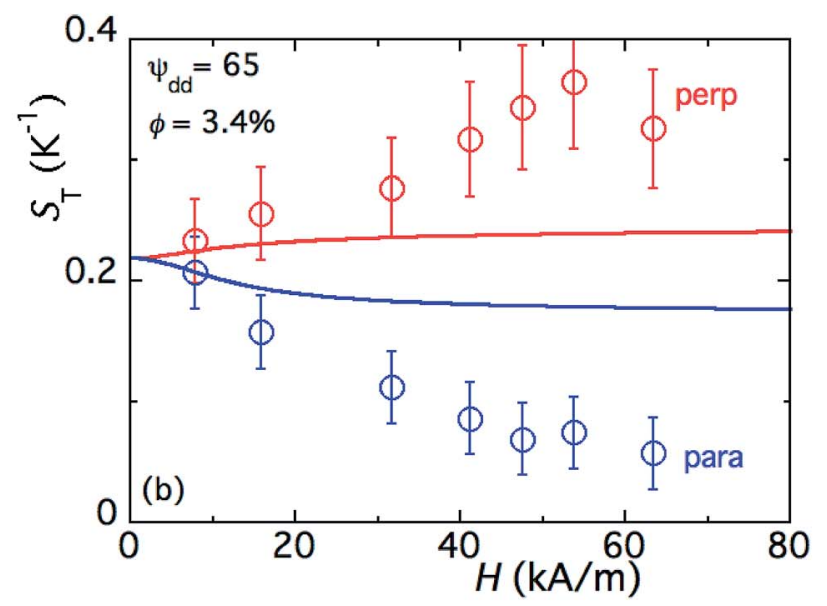

(b)

Fig. 4 (a) Mass diffusion coefficient of FF-DMSO with an NP concentration of $3.4 \%$ as a function of the magnetic field applied perpendicular (blue) and parallel to the temperature gradient. No redox couple added. The solid lines are fits to eqn (12) as a function of the magnetic field. (b) Soret coefficient as a function of the magnetic field applied perpendicular (blue) and parallel (red) to the temperature gradient in FF-DMSO. The NP volume fraction is $3.4 \%$ with $29 \mathrm{mM}$ $\mathrm{ClO}_{4}{ }^{-}$, without a redox couple. The solid lines are fits to eqn (19) as a function of the magnetic field.

the effective volume fraction $\phi_{\text {eff }}$ values are determined to be $8.6 \times 10^{-12} \pm 0.3 \times 10^{-12} \mathrm{~m}^{2} \mathrm{~s}^{-1}$ and $3 \phi$. By fitting eqn (20) and (19) to $S_{\mathrm{T}}(\phi) \ddagger$ (Fig. 3(b)) with the dynamical effective charge number $\xi_{\mathrm{NP}}^{0}=30$, the NP's Eastman entropy of transfer at the infinite dilution limit is determined as $\hat{S}_{\mathrm{NP}}^{0} / k T=1.9 \mathrm{~K}^{-1}$.

4.1.1 Magnetic field dependence. The dipolar interaction parameter $\psi_{\mathrm{dd}}=65$ is deduced from eqn (12) fitted to the experimental diffusion data as a function of $H$ (Fig. 4(a), obtained on a ferrofluid sample with $\phi=3.44 \%)$. $\S$ This parameter can be used to analyse the behaviour of in-field Seebeck coefficients as described in the previous section.

$\ddagger$ Here, $S_{\mathrm{T}}(\phi, H=0)=\chi\left(\phi_{\text {eff }}\right)\left({\widehat{S^{0}}}_{\mathrm{NP}}-\xi_{\mathrm{NP}}^{0} e \mathrm{Se}\right) / k T$ is positive. It is a decreasing function of $\phi$ as $\chi\left(\phi_{\text {eff }}\right)$ decreases with $\phi$ because the interparticle interaction is repulsive $(A 2>0)$. See ref. 44 and 49 .

$\S$ For fitting methods, see Bacri et al..$^{3-36}$
As expected, $S_{\mathrm{T}}(H)$ was found to decrease from its zero-field value (in Fig. 4(b)) when a magnetic field is applied in the direction parallel to the temperature gradient (as much as 70\% at $60 \mathrm{kA} \mathrm{m}^{-1}$ ). Under the perpendicular configuration, on the other hand, $S_{\mathrm{T}}(H)$ increases by $60 \%$ with respect to the zero-field value at $60 \mathrm{kA} \mathrm{m}^{-1}$. While the anisotropic dependency of the $S_{\mathrm{T}}$ response to applied magnetic fields is in agreement with previous reports, ${ }^{24}$ its magnitude is much larger than the theoretical prediction in both field directions $\uparrow$ (as depicted by solid lines in Fig. 4(b)). The large changes observed here are due to the combined effect of the uniform magnetic field and the presence of magnetoconvection. Indeed, our experimental condition $\alpha / \Lambda=1.1$ is within the regime where microconvective instability occurs, driven by the internal demagnetising field (due to the local inhomogeneity in the NP concentration distribution). ${ }^{50,51}$

\subsection{Seebeck coefficient}

In the absence of magnetic nanoparticles, $\mathrm{Se}^{\mathrm{ini}}$ is found negative as was previously reported by Tsierkezos in a large range of non-aqueous solvents. ${ }^{52}$ In order to verify possible dependence of our experimental components on applied magnetic fields (the thermometer readings, the electronic circuitry, the redox couple potential, etc.), both $\mathrm{Se}^{\mathrm{ini}}$ and $\mathrm{Se}^{\mathrm{st}}$ were measured in a reference DMSO solution without magnetic nanoparticles at two different values of $H$. The results show that up to $360 \mathrm{kA} \mathrm{m}^{-1}$, a homogeneous perpendicular magnetic field has no discernible effect on both Seebeck coefficients (data not shown). Therefore, the subsequent magnetic field induced changes in the Seebeck coefficients (initial- and steady-state) presented in this study can be safely attributed to the presence of magnetic nanoparticles in the ferrofluids.

4.2.1 Initial Seebeck coefficients as a function of $\mathrm{H}, \mathrm{Se}^{\mathrm{i}-}$ $\mathbf{n i}(\boldsymbol{H})$. With the experimentally determined parameters (i.e., $D_{\mathrm{NP}}^{0}, \xi_{\mathrm{NP}}^{0}, \hat{S}_{\mathrm{NP}}^{0}$ and $\left.\Psi_{\mathrm{dd}}\right)$ from thermodiffusion measurements at hand, one can now predict the variation of the initial Seebeck coefficient $\Delta \operatorname{Se}^{\text {ini }}(H)=\operatorname{Se}^{\text {ini }}(\phi, H)-\operatorname{Se}^{\text {ini }}(\phi, 0)$ under applied perpendicular magnetic fields through eqn (17). The resulting theoretical curves of $\Delta \mathrm{Se}^{\mathrm{ini}}(H)$ are presented in Fig. 5(a). As expected from having a positive $z_{\mathrm{NP}}$ value, $\Delta \mathrm{Se}^{\mathrm{ini}}$ is also positive, i.e., the absolute value of $\mathrm{Se}^{\mathrm{ini}}$ diminishes. However, the expected magnitude of the change $\Delta \operatorname{Se}^{\mathrm{ini}}(H)$ here is only of the order of $0.1 \mu \mathrm{V} \mathrm{K}^{-1}$ at $H<400 \mathrm{kA} \mathrm{m}^{-1}$, two orders of magnitude below the experimental uncertainty level $\left(\sim 10 \mu \mathrm{V} \mathrm{K}^{-1}\right)$. Therefore, one would not expect to observe the effect of the magnetic field in $\mathrm{Se}^{\text {ini }}$.

Much to our surprise, the experimentally measured field dependence of initial Seebeck coefficients (with $\Delta T=10 \mathrm{~K}$ and the thermocell mean temperature $T=25{ }^{\circ} \mathrm{C}$ ) shows a very different behavior from the theoretical prediction (see Fig. 5(b)). As can be seen from the graph, $\mathrm{Se}^{\mathrm{ini}}(\phi=0.28 \%, H)$ shows only a minor decrease in its absolute value (red symbols), and thus is

I Note that a quantitative agreement was found between the theoretical prediction of $S_{\mathrm{T}}(H)$ and the experimental findings from ref. 24. 


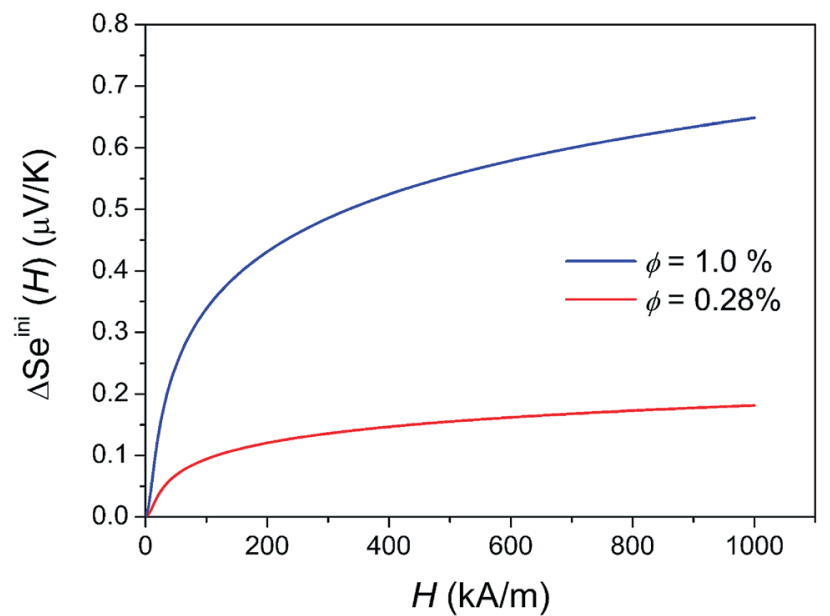

(a)

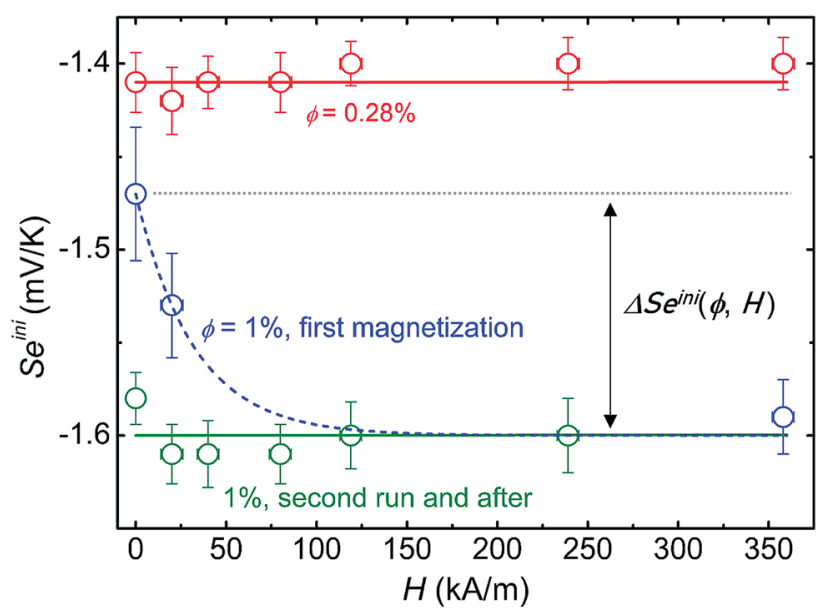

(b)

Fig. 5 (a) Theoretical prediction of initial state Seebeck coefficients as a function of a perpendicularly applied magnetic field $\operatorname{Se}^{\mathrm{ini}}(\phi, H)-$ $\operatorname{Se}^{\mathrm{ini}}(\phi, 0)$ for $\phi=0.28 \%$ and $\phi=1 \%$ according to eqn (17) with experimentally determined $D_{\mathrm{NP}}^{0}, \xi_{\mathrm{NP}}^{0}, \hat{S}_{\mathrm{NP}}^{0}$ and $\Psi_{\mathrm{dd}}$. (b) Experimentally measured $\mathrm{Se}^{\mathrm{ini}}(H)$ for ferrofluids with $\phi=0.28$ and $1 \%$. The error bars correspond to twice the standard error (95\% confidence interval). The red and green solid lines are fits to eqn (1) (i.e. eqn (17) $\left.+\mathrm{Se}^{\mathrm{ini}}(0)\right)$ for $\phi=$ $0.28 \%$ and $\phi=1 \%$ (see Fig. 5(a)). The dashed blue line is a guide to the eye based on an exponential fit, i.e., $y=a+b e^{\frac{x}{c}}$. The field induced change in the initial Seebeck coefficient, $\Delta \operatorname{Se}^{i n i}(\phi, H)$, is indicated with a double-headed arrow.

consistent with the theoretical expectation. || The absolute value of $\operatorname{Se}^{\text {ini }}(\phi=1 \%, H)$ during the first magnetisation (blue symbols), on the other hand, increases by roughly $10 \%$ at $360 \mathrm{kA}$ $\mathrm{m}^{-1}$. This variation of the order of $150 \mu \mathrm{V} \mathrm{K}{ }^{-1}$ is three orders of magnitude larger than the theoretical one $\left(0.1 \mu \mathrm{V} \mathrm{K}^{-1}\right)$ and carries the wrong sign (Fig. 5).

Furthermore, irreversibility is observed in $\operatorname{Se}^{\text {ini }}(H)$ between the first magnetisation and the subsequent measurements (Fig. 5(b), blue and green curves). As can be seen from the

|| These measurements have been performed several times with increasing and decreasing $H$ and the results are reproducible.

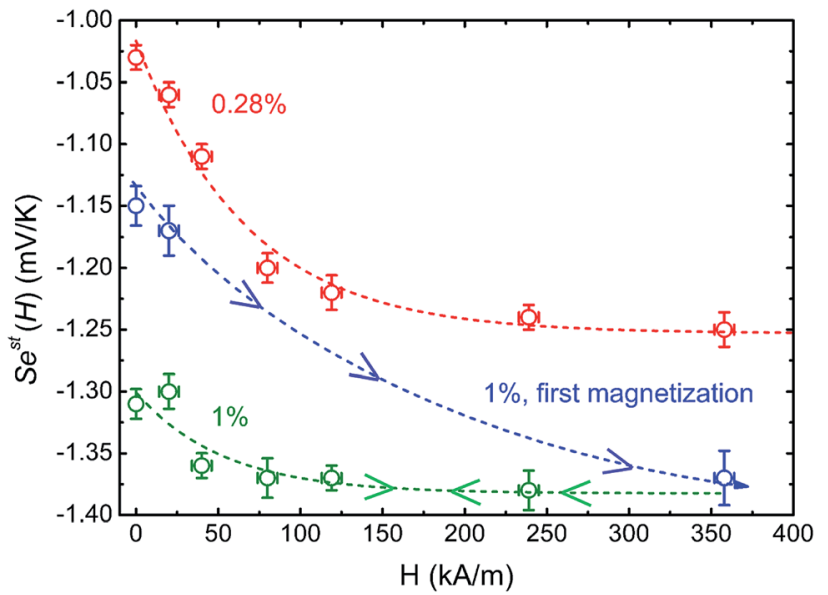

Fig. 6 Apparent steady state Seebeck coefficient as a function of the applied magnetic field $(H)$ for $\phi=0.28 \%$ and $\phi=1 \%$. The red, blue and green dashed lines are guides to the eye based on the exponential, i.e., $y=a+b e^{\underline{x}}$

graph, once the highest magnetic field was reached for the first time, $\mathrm{Se}^{\mathrm{ini}}$ becomes nearly independent of $H$ strength (compatible with the theoretical model). The observed hysteresis suggests that certain irreversible process(es) has taken place during the first magnetisation of the ferrofluid at $\phi=1 \%$. As we will see in more detail below, this phenomenon is accompanied by an increase in the characteristic time to reach an apparent steady state, from $\sim 4.2$ hours during the first magnetisation to $\sim 5.6$ hours for all subsequent measurements (see Fig. 7(a)). Such slowing-down of the NP motion can be explained by the formation of particle aggregates under a strong magnetic field.

To verify this hypothesis, we have post-examined the ferrofluid samples after the in-field Seebeck coefficient measurements via magnetisation and diffusion light scattering (DLS, Vasco de Cordouan Technologies) measurements to search for possible aggregations. The superparamagnetic blocking temperature values $T_{\mathrm{B}}$ determined from the magnetisation measurements (CRYOGENIC SQUID magnetometer, model S700 was used) are $\approx 60 \mathrm{~K}$ for the $\phi=0.28 \%$ sample and $\approx 90 \mathrm{~K}$ for the $\phi=1 \%$ sample. Knowing that $T_{\mathrm{B}}$ increases approximately linearly with the mean NP volume, ${ }^{53}$ this indicates a $\sim 50 \%$ mean volume increase in the more concentrated sample.** The DLS measurements lead to a similar conclusion, with an $\sim 80 \%$ increase in the NPs' hydrodynamic diameter, i.e., an $\sim 600 \%$ increase in the hydrodynamic volume. These two independent measurements confirm that an irreversible nanoparticle aggregation had taken place in the $\phi=1 \%$ sample during the first magnetisation of the Seebeck coefficient measurements. The absence of aggregation in the $\phi=0.28 \%$ sample can be explained by a greater interparticle distance between NPs. As stated earlier, however, such an aggregation phenomenon was not observed during the in-field FRS

** The dipole-dipole interaction energies are negligible at such low concentrations. 


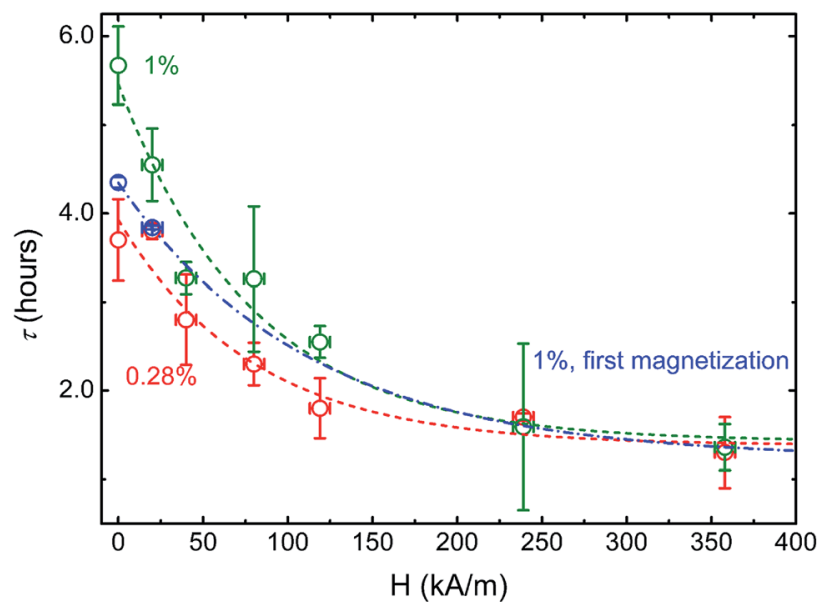

(a)

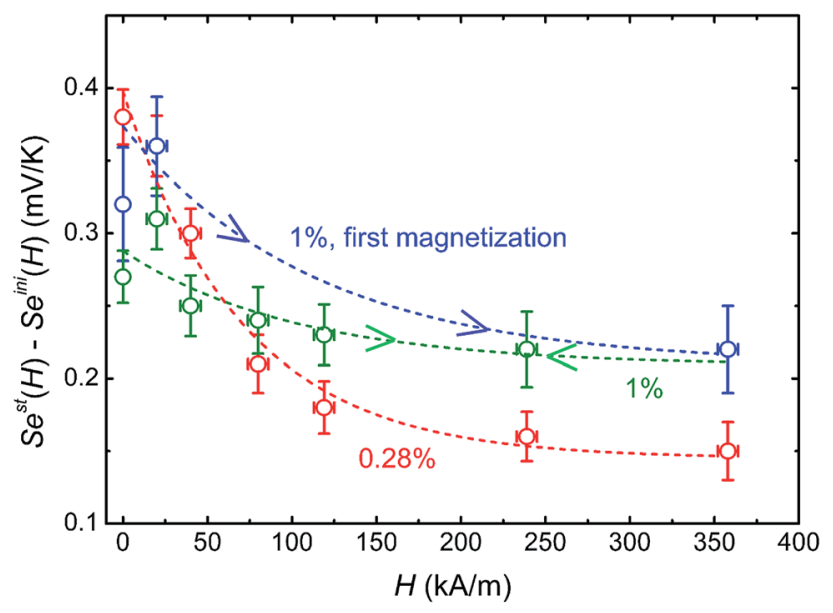

(b)

Fig. 7 (a) Experimentally determined characteristic time to reach the apparent steady state as a function of the applied magnetic field for $\phi$ $=0.28 \%$ and $\phi=1 \%$. (b) Difference between the initial and apparent steady state Seebeck coefficients. The higher the magnetic field, the smaller the difference between the two. In both figures, the error bars correspond to twice the standard error (95\% confidence interval). In both figures, the red, blue and green dashed lines are guides to the eye based on exponential fits i.e., $y=a+b e^{\underline{x}}$.

measurements performed up to $80 \mathrm{kA} \mathrm{m}^{-1}$ even though a higher NP concentration $(\phi=3.44 \%)$ was used. The two most significant differences between the two experiments are (i) the absence (presence) of redox couple agents and (ii) the total time duration for which the ferrofluid sample is exposed to the external magnetic field, i.e., one (several) hour in the thermodiffusion (thermoelectric) measurements, respectively. Thus, it is likely that the modification of inter-particle electrostatic force due to the presence of redox couple molecules and the longer experimental time-scale both contribute to the formation of NP aggregates.

It should also be noted that $\left|\mathrm{Se}^{\text {ini }}\right|$ increases by approximately $10 \%$ after the formation of particle aggregates (Fig. 5(b), green curve). These aggregates appear to be stable, i.e., the infield Seebeck measurements are reproducible after the first magnetisation. The physical and/or chemical origins behind this phenomenon are far from trivial. However, within the framework of existing theories, possible explanations include an increase in the Eastman entropy of transfer of the dispersed objects and the simultaneous reduction of $n$ (NP number) at a constant $\phi$, both due to the aggregation. The latter can also indirectly influence the thermogalvanic term in eqn (1) via reshaping the ionic environment surrounding the redox couple molecules leading to modifications in the standard reaction entropies of redox reactions at the electrodes. ${ }^{54}$

4.2.2 Seebeck coefficient as a function of $\boldsymbol{H}$ in the apparent steady state, $\operatorname{Se}^{\text {st }}(\boldsymbol{H})$. The apparent steady state Seebeck coefficients as a function of magnetic field, $\operatorname{Se}^{\mathrm{st}}(H)$, for both ferrofluids are presented in Fig. 6. An increase of $\sim 25 \%$ in $\left|\mathrm{Se}^{\mathrm{st}}\right|$ is observed for $\phi=0.28 \%$, which is reproducible after repeated magnetisation-demagnetisation cycles. The $\sim 13 \%$ increase for $\phi=1 \%$, however, is only present during the first magnetisation and after which $\mathrm{Se}^{\text {st }}(H)$ becomes irreversible. During the subsequent magnetisation-demagnetisation cycles, $\operatorname{Se}^{\text {st }}(\mathrm{H})$ shows a reduced (but stable and reproducible) field dependency. It is worth noting that for both samples (red and green curves) $\mathrm{Se}^{\mathrm{st}}(H)$ saturates around $100 \mathrm{kA} \mathrm{m}^{-1}$, a magnetic field that can be easily attained with permanent magnets, and thus promising for potential applications.

4.2.3 Time constant. We now shift our focus to the characteristic time constants, $\tau$, required to reach the apparent steady state under magnetic fields. Fig. 7(a) presents the time constants required to reach the apparent steady state as a function of $H$ for both ferrofluids (deduced from an exponential fit to the measured Seebeck voltage values). These results are highly reproducible under field-cycling as shown in Fig. 8.

In a zero-field, the apparent steady state is reached in $\tau$ less than 6 hours for all samples. However, the characteristic times $\tau^{\mathrm{Eq}}$ to reach the Soret equilibrium can be estimated $v i a \tau^{\mathrm{Eq}}=l^{2} /$ $\left(\pi^{2} D_{\mathrm{NP}}{ }^{2}\right.$ ) (where $l=6 \mathrm{~mm}$ is the diffusion length, i.e., the distance between the two electrodes, and $D \approx 1.2 \times 10^{-11} \mathrm{~m}^{2}$ $\mathrm{s}^{-1}$ at $\phi=1 \%$, see Fig. 3(a)). This gives $\tau^{\mathrm{Eq}} \approx 84$ and $\approx 100$

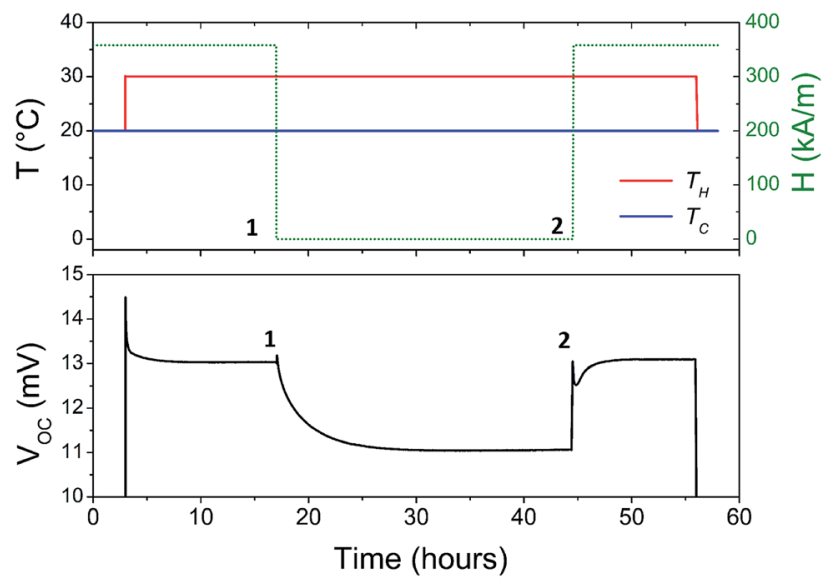

Fig. 8 Variation of apparent steady-state Seebeck voltage in the $\phi=$ $0.28 \%$ sample. The magnetic field is decreased from $360 \mathrm{kA} \mathrm{m}^{-1}$ to $0 \mathrm{kA} \mathrm{m}^{-1}$ (point 1) and increased again (point 2) while maintaining $\Delta T=$ $10 \mathrm{~K}$. The results are reproducible. 
hours for $\phi=1$ and $0.28 \%$, respectively, more than one order of magnitude larger than the experimentally determined $\tau$ values. Such a large discrepancy suggests that the observed apparent steady state is established due to a physical phenomenon different from the Soret equilibrium. A similar observation has already been made recently in aqueous ferrofluids. ${ }^{18}$ It was suggested that the apparent steady state stems from a temperature dependent NP adsorption and/or ordering phenomenon occurring at the electrode-fluid interfaces, which stabilizes much quicker than the Soret equilibrium. The NP adsorption has indeed been observed on mercury, ${ }^{55}$ gold $^{56}$ and platinum ${ }^{57}$ electrodes in aqueous ferrofluids, and the ordering phenomenon has been reported on $\mathrm{SiO}_{2}$ surfaces. ${ }^{58}$ The electrostatic interactions (between the surface, the particles and the counterions) and inter-particle magnetic interactions (at high MNP concentrations) as well as the surface geometry (undulations and channels ${ }^{59}$ ) are known to contribute to such phenomena, creating a surface-stabilized layer of NPs with much higher concentration of NPs than that of the bulk ferrofluid. Increased concentration of charged NPs can then modify the local ionic strength at the electrode-fluid interface, and therefore the redox reaction entropy term in both $\mathrm{Se}^{\text {ini }}$ and $\mathrm{Se}^{\text {st }}$ (eqn (1) and (18)).

Under an applied magnetic field, marked reductions in both $\tau(H)$ and $\mathrm{Se}^{\mathrm{st}}(H)-\mathrm{Se}^{\mathrm{ini}}(H)$, by a factor of 2 to 4 , are observed between 0 and $360 \mathrm{kA} \mathrm{m}^{-1}$ (as presented in Fig. 7(a) and (b), respectively) as if the presence of the magnetic field attenuates the NP adsorption and/or layering on the electrode surface. Insight into the NP adsorption/layering phenomenon can be gained from the Molecular Dynamics simulation by Jordanovic and Klapp ${ }^{60}$ where they have shown that the application of a magnetic field in the direction parallel to the ferrofluidsubstrate interface can destroy NP layers. When a sufficiently high magnetic field is applied, the magnetic nanoparticles tend to align themselves along the external field (chain formation) due to their superparamagnetic nature. This leads to repulsive dipolar interactions between neighboring chains in the direction perpendicular to the electrode surface $\dagger^{\dagger} \dagger$ and thus limits the number of adsorbed NPs. Although the numerical simulations cited $^{60}$ were performed for ferrofluids with high NP concentration values ( $\phi=20 \%$ and higher) and with a nonconducting substrate, the surface-initiated layering of dipolar particles and their field dependence are considered to be generic features of confined dipolar liquids. Consequently, the NP distribution near the electrode surface at the apparent steady state under a magnetic field remains closer to that of the initial state than that of the zero-field. Consequently, the redox reaction entropy contribution to the Seebeck coefficient also remains similar between the two states as depicted in Fig. 7(b). The effect of the magnetic field on the Seebeck voltage and its time evolution are clearly visible in Fig. 8. When the magnetic field of $350 \mathrm{kA} \mathrm{m}^{-1}$ is turned off after the thermocell has reached its apparent steady state (point 1 , dotted green curve),

† The magnetic interaction is attractive for particles aligned one-behind-another; however, it is repulsive in the direction perpendicular to $H$, expanding the NP distribution near the electrodes. the Seebeck voltage decreases as more NPs adsorb with a characteristic time $\tau \sim 4$ hours. The latter corresponds to the time constant recorded at $0 \mathrm{kA} \mathrm{m}^{-1}$. When $H=360 \mathrm{kA} \mathrm{m}^{-1}$ is applied again, the Seebeck voltage increases back quickly, presumably due to the quick ejection of NPs.

\section{Conclusion}

The Seebeck coefficient of DMSO-based dilute ferrofluids was examined under the influence of an external magnetic field. The magnetic field was applied perpendicularly to the temperature gradient, the configuration under which a marked increase in Soret coefficients was observed. The Seebeck coefficient was found to increase by as much as $25 \%$ with a moderate magnetic field strength of $100 \mathrm{kA} \mathrm{m}^{-1}$; however, the subsequent analysis showed that the observed phenomena cannot be explained by the existing theoretical model which takes into account the local magnetic field gradient induced by the magneto-thermodiffusion of nanoparticles. Plausible physical and physicochemical origins leading to the enhancements of the fluids' Seebeck coefficient include the temperature and magnetic fielddependent auto-organisation of NPs at the electrode surface and its ramification on the thermogalvanic potential of redox couples.

To the best of our knowledge, this work presents the first evidence of thermopower enhancement induced by the application of a magnetic field. Only a moderate magnetic field strength of about $100 \mathrm{kA} \mathrm{m}^{-1}$ (less than $0.2 \mathrm{~T}$ ) is needed to increase the Seebeck coefficient, easily attainable with a strong permanent magnet. The enhancement effect is more pronounced at lower nanoparticle concentration $(0.28 \%)$, while at a higher concentration (1\%) the use of a high magnetic field led to an irreversible aggregation of nanoparticles. Thus, dilute ferrofluids made with more conducting electrolytes such as ionic liquids should be considered for the next step toward the application of ferrofluids in magneto-thermoelectric technology.

\section{Conflicts of interest}

There are no conflicts to declare.

\section{Acknowledgements}

This work received financial support from ANR TEFLIC (Grant No. ANR-12-PRGE-0011-01), LABEX-PALM (Grant No. ANR-10LABX-0039-PALM), Program CAPES-COFECUB no. 714/11 (France-Brazil) and the European Union's Horizon 2020 research and innovation programme under grant agreement no. 731976 (MAGENTA).

\section{References}

1 M. H. Elsheikh, D. A. Shnawah, M. F. M. Sabri, S. B. M. Said, M. H. Hassan, M. B. A. Bashir and M. Mohamad, Renewable Sustainable Energy Rev., 2014, 30, 337-355. 
2 M. W. Gaultois, T. D. Sparks, C. K. H. Borg, R. Seshadri, W. D. Bonificio and D. R. Clarke, Chem. Mater., 2013, 25, 2911-2920.

3 E. Vanecht, K. Binnemans, S. Patskovsky, M. Meunier, J. W. Seo, L. Stappers and J. Fransaer, Phys. Chem. Chem. Phys., 2012, 14, 5662-5671.

4 S. Choi, ASME Fluids Eng. Div., 1995, 231, 99.

5 J. Eastman, S. R. Phillpot, S. U. S. Choi and P. Keblinski, Annu. Rev. Mater. Res., 2004, 34, 219-246.

6 T. Ambreen and M.-H. Kim, Renewable Sustainable Energy Rev., 2018, 91, 564-583.

7 M. J. Assael, K. D. Antoniadis, W. A. Wakeham and X. Zhang, Int. J. Heat Mass Transfer, 2019, 138, 597-607.

8 D. Kumar, D. Valan and A. Amirtham, Renewable Sustainable Energy Rev., 2016, 60, 21-40.

9 C.-C. Lai, W.-C. Chang, W.-L. Hu, Z. M. Wang, M.-C. Lu and Y.-L. Chueh, Nanoscale, 2014, 6, 4555.

10 O. Mahian, A. Kianifar, S. A. Kalogirou, I. Pop and S. Wongwises, Int. J. Heat Mass Transfer, 2013, 57, 582.

11 A. Kasaeian, A. T. Eshghi and M. Sameti, Renewable Sustainable Energy Rev., 2015, 43, 584-598.

12 A. Würger, Rep. Prog. Phys., 2010, 73, 126601.

13 I. P. J. Burelbach, D. Frenkel and E. Elser, Eur. Phys. J. E, 2018, 41, 7.

14 S. Di Lecce and F. Bresme, J. Phys. Chem. B, 2018, 122, 16621668.

15 R. F. Stout and A. S. Khair, Phys. Rev. E, 2017, 96, 022604.

16 S. Duhr and D. Braun, Proc. Natl. Acad. Sci., 2006, 103, 19678-19682.

17 S. A. Putnam and D. G. Cahill, Langmuir, 2005, 21, 53175323.

18 T. J. Salez, B. T. Huang, M. Rietjens, M. Bonetti, C. WiertelGasquet, M. Roger, C. L. Filomeno, E. Dubois, R. Perzynski and S. Nakamae, Phys. Chem. Chem. Phys., 2017, 19, 9409-9416.

19 B. T. Huang, M. Roger, M. Bonetti, T. J. Salez, C. WiertelGasquet, E. Dubois, R. Cabreira Gomes, G. Demouchy, G. Mériguet, V. Peyre, M. Kouyaté, C. L. Filomeno, J. Depeyrot, F. A. Tourinho, R. Perzynski and S. Nakamae, J. Chem. Phys., 2015, 143, 054902.

20 M. F. Dupont, D. R. MacFarlane and J. M. Pringle, Chem. Commun., 2017, 53, 6288-6302.

21 E. Blums, J. Magn. Magn. Mater., 2005, 289, 246-249.

22 E. Blums, S. Odenbach, A. Mezulis and M. Maiorov, Phys. Fluids, 1998, 10, 2155-2163.

23 T. Voelker and S. Odenbach, Phys. Fluids, 2005, 17, 037104.

24 M. Kouyaté, C. L. Filomeno, G. Demouchy, G. Mériguet, S. Nakamae, V. Peyre, M. Roger, A. Cébers, J. Depeyrot, E. Dubois and R. Perzynski, Phys. Chem. Chem. Phys., 2019, 21, 1895-1903.

25 T. J. Salez, M. Roger, R. Perzynski, A. Cebers and S. Nakamae, Entropy, 2018, 20, 405.

26 W. Nernst, Z. Phys. Chem., 1889, 4, 129-181.

27 A. Bund, S. Koehler, H. Kuehnlein and W. Plieth, Electrochim. Acta, 2003, 49, 147-152.

28 S. R. Ragsdale, K. M. Grant and H. S. White, J. Am. Chem. Soc., 1998, 120, 13461-13468.
29 D. T. Kountouras, C. A. Vogiatzis, A. Tsouknidas and S. Skolianos, Corros. Eng., Sci. Technol., 2014, 49, 603607.

30 C. L. Filomeno, M. Kouyaté, V. Peyre, G. Demouchy, A. F. C. Campos, R. Perzynski, F. A. Tourinho and E. Dubois, J. Phys. Chem. C, 2017, 121, 5539-5550.

31 N. F. Carnahan and K. E. Starling, J. Chem. Phys., 1969, 51, 635-636.

32 E. Blums, A. Cebers and M. M. Maiorov, Magnetic Fluids, Walter de Gruyter, 1997.

33 C. Kittel, Physique de l'état solide - 8eme édition, Dunod, 2007.

34 J. C. Bacri, A. Cebers, A. Bourdon, G. Demouchy, B. M. Heegaard and R. Perzynski, Phys. Rev. Lett., 1995, 74, 5032-5035.

35 F. Gazeau, E. Dubois, J.-C. Bacri, F. Boué, A. Cebers and R. Perzynski, Phys. Rev. E, 2002, 65, 031403.

36 G. Mériguet, F. Cousin, E. Dubois, F. Boué, A. Cebers, B. Farago and R. Perzynski, J. Phys. Chem. B, 2006, 110, 4378-4386.

37 E. Wandersman, E. Dubois, F. Cousin, V. Dupuis, G. Mériguet, R. Perzynski and A. Cebers, Europhys. Lett., 2009, 86, 10005.

38 G. Mériguet, M. Jardat and P. Turq, J. Chem. Phys., 2005, 123, 144915.

39 G. Mériguet, E. Dubois, M. Jardat, A. Bourdon, G. Demouchy, V. Dupuis, B. Farago, R. Perzynski and P. Turq, J. Phys.: Condens. Matter, 2006, 18, S2685.

40 V. Zinovyeva, S. Nakamae, M. Bonetti and M. Roger, ChemElectroChem, 2014, 1, 426-430.

41 J. N. Agar and J. C. R. Turner, Proc. R. Soc. London, Ser. A, 1960, 255, 307-330.

42 A. Würger, Phys. Rev. Lett., 2008, 101, 108302.

43 A. Majee, PhD thesis, Université Bordeaux I, 2012.

44 R. Cabreira Gomes, A. Ferreira da Silva, M. Kouyaté, G. Demouchy, R. Aquino, E. Dubois, S. Nakamae, J. Depeyrot, M. Roger and R. Perzynski, Phys. Chem. Chem. Phys., 2018, 20, 16402.

45 R. Massart, IEEE Trans. Magn., 1981, 17, 1247-1248.

46 G. Demouchy, A. Mezulis, A. Bee, D. Talbot, J. C. Bacri and A. Bourdon, J. Phys. D: Appl. Phys., 2004, 37, 1417.

47 J. C. Bacri, R. Perzynski, D. Salin, V. Cabuil and R. Massart, J. Magn. Magn. Mater., 1990, 85, 27-32.

48 M. Bonetti, S. Nakamae, B. T. Huang, T. J. Salez, C. WiertelGasquet and M. Roger, J. Chem. Phys., 2015, 142, 244708.

49 M. Kouyaté, C. Filomeno, G. Demouchy, G. Mériguet, S. Nakamae, V. Peyre, M. Roger, A. Cebers, J. Depeyrot, E. Dubois and R. Perzynski, Phys. Chem. Chem. Phys., 2019, 21, 1895-1903.

50 D. Zablotsky, A. Mezulis and E. Blums, C. R. Mec., 2013, 341, 449-454.

51 D. Zablotsky, PhD thesis, University of Latvia - Riga, 2012.

52 N. Tsierkezos, J. Solution Chem., 2007, 36, 289-302.

53 D. F. J. L. Doemann and E. Tronc, Adv. Chem. Phys., 1997, XCVIII, 283-494. 
54 B. Huang, S. Muy, S. Feng, Y. Katayama, Y.-C. Lu, G. Cheng and Y. Shao-Horn, Phys. Chem. Chem. Phys., 2018, 20, 15680-15686.

55 E. Dubois and J. Chevalet, Langmuir, 2003, 19, 10892-10900.

56 I. T. Lucas, E. Dubois, J. Chevalet and S. Durand-Vidal, Phys. Chem. Chem. Phys., 2008, 10, 3263-3273.

57 T. J. Salez, PhD thesis, Université de recherche Paris Sciences et Lettres, École Normale Supérieure, 2018.
58 A. Vorobiev, J. Major, H. Dosch, G. Gordeev and D. Orlova, Phys. Rev. Lett., 2004, 93, 267203.

59 C. Y. Matuo, A. Bourdon, A. Bee and A. M. F. Neto, Phys. Rev. $E$, 1997, 56, R1310-R1313.

60 J. Jordanovic and S. H. L. Klapp, Phys. Rev. Lett., 2008, 101, 038302. 